\title{
A Coherent Spurious Analysis for sub-THz Frequency Multiplier Chains
}

\author{
Mostafa Jafari Nokandi, Aarno Pärssinen, Timo Rahkonen \\ Circuits and Systems Research Unit (CAS) \\ ITEE Faculty, University of Oulu \\ Oulu, Finland \\ mostafa.jafarinokandi@oulu.fi, aarno.parssinen@oulu.fi,timo.rahkonen@oulu.fi
}

\begin{abstract}
At sub-THz frequencies, the local oscillation for transceivers is often generated by an analog frequency multiplier tree. This paper studies the origins and minimization of spurious tones in a local oscillator multiplier consisting of a chain of frequency doublers. It is found that the nearest spurious components depend on the leakage of the input signal in the first multiplier, which can be minimized by active bias tuning, filtering in the first stage or better balance in the driving differential signal. To verify the nonlinearity modeling, 0.13- $\mu \mathrm{m}$ SiGe BiCMOS technology has been utilized.
\end{abstract}

Index Terms-Spurious, Frequency Multiplier, Non-linear system, Mismatch

\section{INTRODUCTION}

PLL synthesizers play a fundamental role in carrier frequency generation in the $1-3 \mathrm{GHz}$ mobile bands [1]-[3]. Commercial PLL synthesizers are available up to $20-30 \mathrm{GHz}$ [4], but beyond that the PLL type synthesizers and VCOs tend to become overly power-hungry. So, in order to decrease the external reference frequency, the use of various injectionlocking methods or open-loop frequency multiplier circuits with a high ratio of multiplying is inevitable [5]-[7].

Frequency multipliers employ multiplication or nonlinearities to generate higher order harmonics [8], [9]. Ideally, the multiplier should generate just the desired harmonic frequency $n \omega_{o}$, but mismatches and the non-linear nature of the multiplier cause additional spurious tones to be generated. Spurious LO tones cause erroneous mixing in the transceiver. If the spurious tones are near the desired harmonic $\left(n \omega_{o}\right)$, they may cause the signal band to mix with itself. This is illustrated in Fig. 1: if the signal bandwidth BW is larger than the spacing of the LO tones (when $\omega_{o}<\mathrm{BW}$ of data), the bands overlap, reducing SNR. Since having a wide bandwidth is required in sub-THz applications, multiplying by more than $\times 8$ (such as $\times 16$ ) can cause such aliasing [10]. The required spurious rejection (SR) depends on the minimum SNR of the modulation used and can be up to $30 \mathrm{~dB}$ for multi-level QAM modulation.

In this paper, we concentrate on the typical multiply-bytwo circuit as a popular multiplying approach, and analyse the originating mechanisms and propagation of spurious tones

This research has been financially supported by the Academy of Finland 6Genesis Flagship (grant 318927).

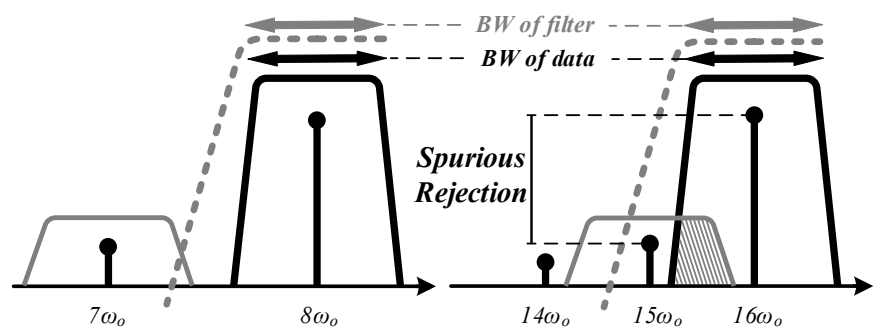

(a)

(b)

Fig. 1. a) Spurious free condition for low ratio $(\times 8)$ of multiplying. b) Corruption of the wanted band due to adjacent spurious tone for high ratio $(\times 16)$ of multiplying.

in a frequency multiplier consisting of a chain of multiply-bytwo circuits. Section II reviews the operation of the multiplyby-two circuit and presents a sufficient behavioral model to describe the non-linearity. Section III models the spurious signal generation and their propagation, and shows the relation between the products of the last stage and the first stage. Section IV gives a solution to suppress the main cause of the nearest spurious tone based on bias tuning. Section V investigates the feasibility of filtering in different stages. Finally, Section VI concludes the causes of spurious tones and the solutions to suppress them.

\section{StRUCTURE AND OPERATION OF THE MULTIPLY-BY-2 CIRCUIT}

Fig. 2 shows the simplified schematic of the multiply-bytwo circuit [6]. It utilises the even order non-linearity of the transistors to generate an even harmonic of the input frequency. Assuming a fully differential input drive, the differential linear and odd-order distortion terms will be cancelled when summing the transistor outputs, but even-order distortion appears as a common-mode signal and will be coherently summed into the output. The output is now a single-ended signal that needs to be converted to a balanced one before passing to the next stage.

The Taylor series of the exponential $I_{C}-V_{B E}$ function is a common starting point for estimating the strength of the nonlinearity. This suggests that second order analysis is sufficient only up to $V_{i n}=20 \mathrm{mV}$. As seen from the simulated harmonic responses in Fig. 3, in practice the device is linearized by the 


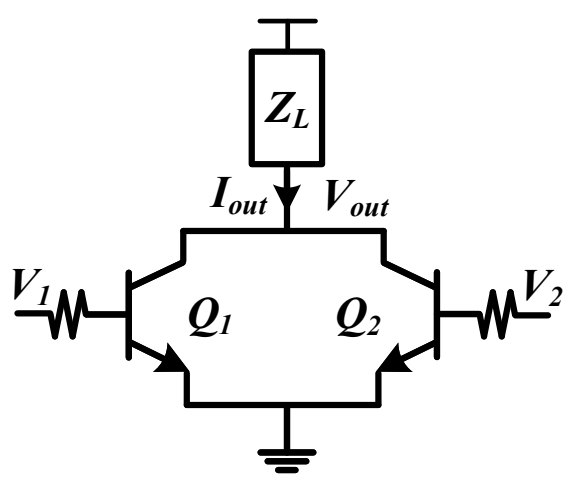

Fig. 2. Simplified schematic of the frequency doubler circuit.

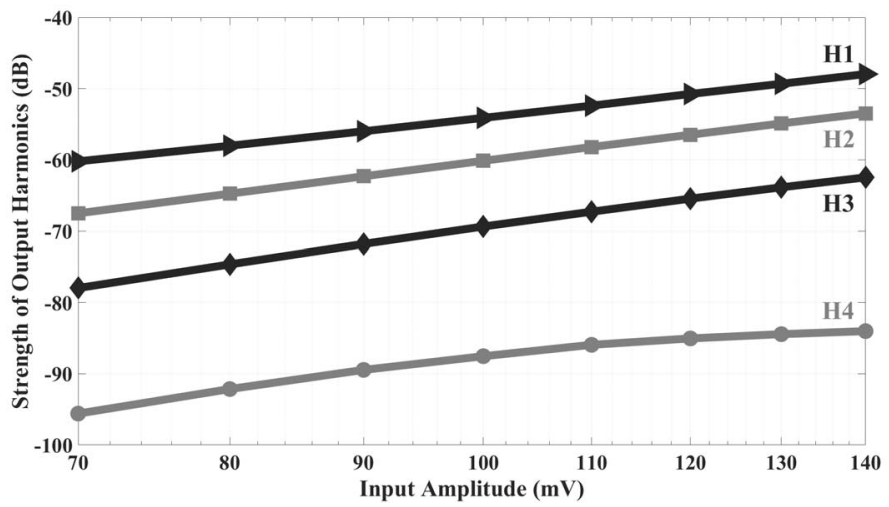

Fig. 3. The amplitude of fundamental and harmonics tones of the output current of a single transistor as a function of input amplitude (The transistor has been biased at $750 \mathrm{mV}$ and the load is $50 \Omega$ ).

parasitics, and the fourth degree non-linearity can be ignored in hand analysis. So, using second-degree models for $I_{C}$ currents in Fig. 2, the output current $I_{\text {out }}$ of the multiplyby- 2 circuit can be modeled by (1), where $K_{0}, K_{1}$ and $K_{2}$ are the DC current, linear gain and second-order non-linearity of a single transistor, respectively. For the transistors used, the numerical values at the chosen bias are roughly $K_{0}=1.22 \mathrm{~mA}$, $K_{1}=0.0115 A / V$ and $K_{2}=0.163 A / V^{2}$.

$$
I_{\text {out }}=2 K_{0}+K_{1}\left(V_{1}+V_{2}\right)+K_{2}\left(V_{1}^{2}+V_{2}^{2}\right)
$$

\section{SPURIOUS GENERATION ANALYSIS}

Fig. 4 shows a more detailed schematic of the multiply-by-2 circuit. Its load circuit can act as both a load and a resonant filter, and the single-ended output needs to be converted to a balanced signal using a balun circuit. Combining the transistor outputs to $I_{\text {out }}$ and using (1), we get the doubled frequency.

It is difficult to make the balun circuit completely symmetrical, since common mode signals caused by mismatch do not get cancelled, but will be amplified into the output. In the multiplier shown in Fig. 4, the main sources of mismatch are amplitude and phase mismatch in the balun. Since the active devices are always placed near each other, the mismatch originated from process variation in the active part can be assumed small. Instead of modeling the output of the balun as

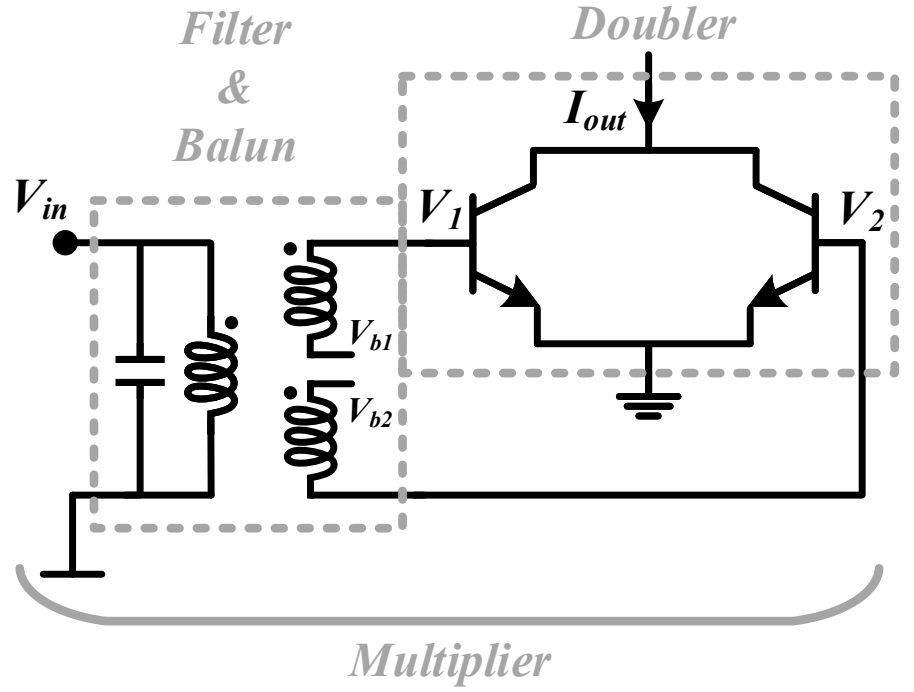

Fig. 4. The multiplier block in detail, including the filter, balun and doubler part.

a combination of common mode and differential mode signals $\left(V_{1}=V_{c}+V_{d}\right.$ and $\left.V_{1}=V_{c}-V_{d}\right)$, the modeling can be simplified by just adding an error signal $e$ in one of the inputs:

$$
\begin{aligned}
& V_{1}=V_{i n} \\
& V_{2}=V_{i n}\left(1+e_{a}\right) e^{j\left(-\pi+e_{p}\right)}
\end{aligned}
$$

Here, $e_{a}$ and $e_{p}$ show the errors due to the amplitude and phase unbalance in the balun, respectively. It will be demonstrated that the existence of these errors in the first stage propagates the spurious tones to the subsequent stages of the multiplier chain as well. As the multiplier chain has no feedback, the phase of any node can be considered as a reference and each stage can be studied independently. Therefore, as shown in Fig. 5, at the $k^{t h}$ multiplier, the input $\left(A_{m}\right)$ can be defined as a combination of $2^{k-1}$ components which illustrate the wanted harmonic and lower spurious tones

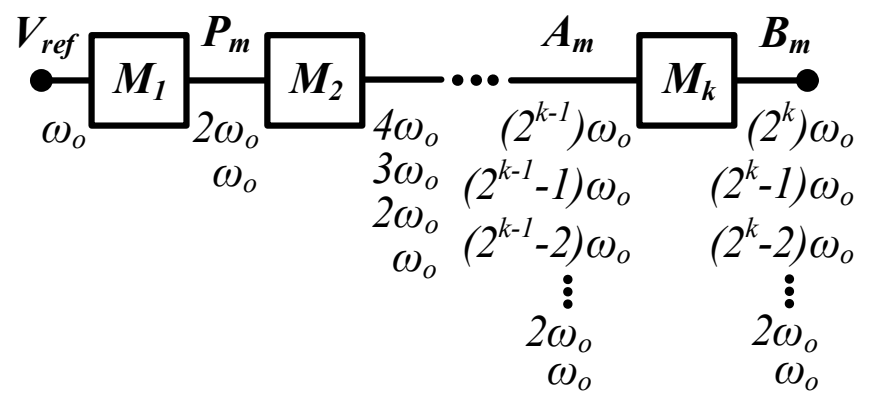

Fig. 5. Propagation of spurious tones through the multiplier chain. $B_{m}, A_{m}$ and $P_{m}$ are the output of $k^{t h},(k-1)^{t h}$ and $1^{s t}$ stages, respectively and $V_{r e f}$ is the reference input of the chain 


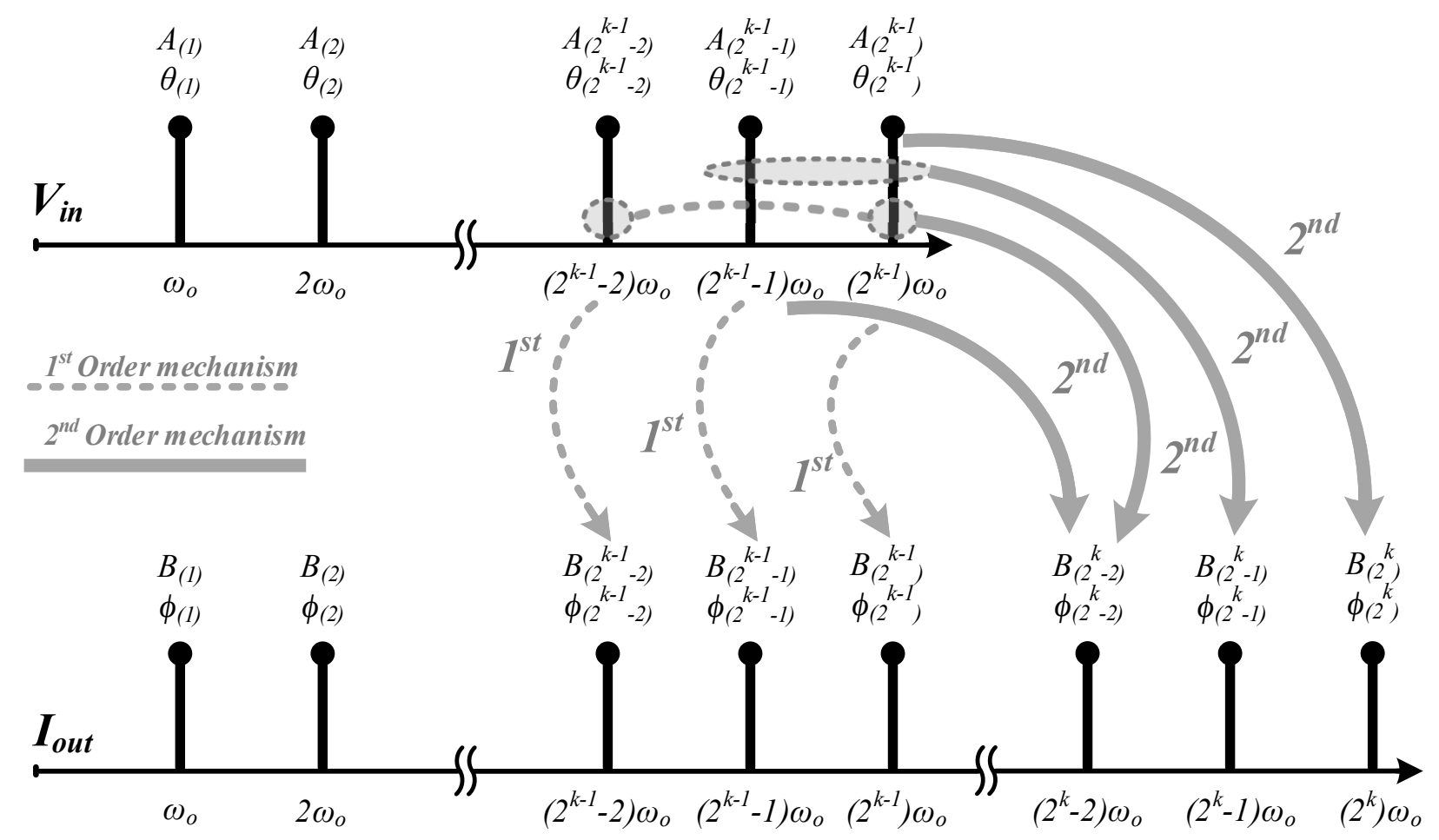

Fig. 6. Generation of nearest spurious tones in $k^{\text {th }}$ stage. A's and B's are input tones and output tones

due to all previous stages as

$$
V_{i n}=\sum_{m=-\frac{n}{2}}^{\frac{n}{2}} A_{m} e^{j \theta_{m}} e^{j m \omega_{o} t}
$$

where $n=2^{k}$ is the amount of multiplication and $A_{m}$ and $\theta_{m}$ are the amplitude and phase of each component. After passing through the filter and balun which are part of the multiplier, $V_{i n}$ experiences both attenuation for lower components due to the bandpass nature of the load and mismatch due to the balun. So, $V_{1}$ and $V_{2}$ will be

$$
\begin{aligned}
& V_{1}=\sum_{m=-\frac{n}{2}}^{\frac{n}{2}} A_{m}^{\prime} e^{j \theta_{m}^{\prime}} e^{j m \omega_{o} t} \quad \text { and } \\
& V_{2}=\sum_{m=-\frac{n}{2}}^{\frac{n}{2}}\left(1+e_{a}\right) A_{m}^{\prime} e^{j\left(-\pi+\theta_{m}^{\prime}+e_{p}\right)} e^{j m \omega_{o} t}
\end{aligned}
$$

where $A_{m}^{\prime}$ and $\theta_{m}^{\prime}$ represent the possible effects of the filter on the amplitude and phase of the input signal. $I_{\text {out }}$ in (1) consists of squares of $V_{1}$ and $V_{2}$, which can be expanded to crossproducts according to (5) when the sum of $C_{m}$ corresponds to $V_{1}$ or $V_{2}$.

$$
\left(\sum_{m=-\frac{n}{2}}^{\frac{n}{2}} C_{m}\right)^{2}=\sum_{i=-\frac{n}{2}}^{\frac{n}{2}} \sum_{j=-\frac{n}{2}}^{\frac{n}{2}} C_{i} C_{j}
$$

All the possible permutations of the binary combinations contribute to the output current. Thus, $I_{\text {out }}$ can be written as:

$$
I_{\text {out }}=\sum_{m=-n}^{n} B_{m} e^{j \phi_{m}} e^{j m \omega_{o} t}
$$

where $B_{m}$ and $\phi_{m}$ are the amplitude and phase of each component at the output. As exhibited in Fig. 6, only $\left(2^{k-1}-\right.$ 1) $\omega_{o}$ and $\left(2^{k-1}\right) \omega_{o}$ participate in the generation of the closest spurious components in the output. Hence, we are essentially interested in two things: how the nearest spurious component gets generated as a sum frequency of the two highest tones in the input, and how the lower tone gets generated in the first place. We can conclude that the mismatch of the input is crucial in the first stage (as it causes the input leakthrough), but after that the behavior is dominated by the square law mixing process.

Using (1), (4) and (6) and considering $e_{a}=e_{p}=0$, (7) is obtained:

$$
\begin{aligned}
\frac{1}{S R}=\frac{B_{\left(2^{k}-1\right)}}{B_{\left(2^{k}\right)}} & =\frac{A_{\left(2^{k-1}\right)}^{\prime} A_{\left(2^{k-1}-1\right)}^{\prime}}{A_{\left(2^{k-1}\right)}^{\prime}}=\frac{P_{(2)}^{\left(2^{k-1}-1\right)} P_{(1)}}{P_{(2)}^{\left(2^{k-1}\right)}} \\
& =\frac{A_{\left(2^{k-1}-1\right)}^{\prime}}{A_{\left(2^{k-1}\right)}^{\prime}}=\frac{P_{(1)}}{P_{(2)}}
\end{aligned}
$$

where $P_{(2)}$ and $P_{(1)}$ are the amplitudes of the desired and spurious outputs of the first multiplier and $S R$ is the desired spurious rejection of the nearest spurs in the output. According to (7), the spurious rejection requirement is equal in all the 


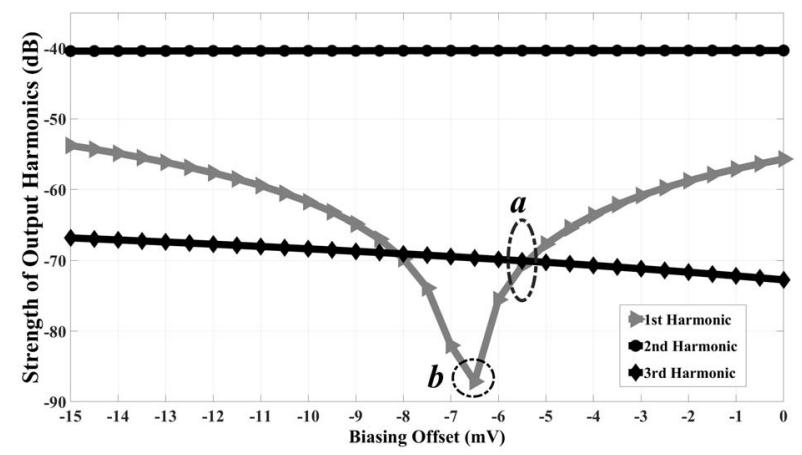

Fig. 7. Changes in the spurious components near the second harmonic in terms of the biasing offset.

stages and can be applied in any. However, it will be easiest in the first stage.

Unlike subsequent stages, the nearest spurious tone in the output of the first stage originates from the linear gain $\left(K_{1}\right)$ and is caused by mismatch in the first balun. This spurious tone can be reduced by improving the matching of the balun, or by filtering the $\omega_{o}$ leakthrough term. However, the effect of the mismatch can also be compensated by adjusting the gains of the two transistors in the multiplier.

\section{Minimizing the LO LEAKAGE By Bias ADJUSTMENT}

As shown above, the LO leakage is caused by mismatched fundamental output currents. Magnitude errors can be compensated by tuning the gains of the transistors in the multiplier, and for this purpose, each transistor is individually biased as shown in Fig. 4. This makes it possible to cancel the fundamental leakage completely while causing a slight increase in the third harmonic leakage at the same time, which imposes a compromise on the designer.

In a simplified simulation shown in Fig. 7, we had $10 \%$ mismatch in the input amplitudes, and two scenarios of bias adjustment were taken into consideration. In the first case, by $-5.5 \mathrm{mV}$ bias adjustment, the LO leakage was reduced by $14 \mathrm{~dB}$ to $-30 \mathrm{dBc}$ level. The value $-5.5 \mathrm{mV}$ was chosen based on the point in which the spurious components at both sides of the desired harmonic are equal, as illustrated in Fig. 7. The problem with this case is that there are still spurious components of $-30 \mathrm{dBc}$ both at $\omega_{o}$ and $3 \omega_{o}$. Hence, another case $(-6.5 \mathrm{mV})$ can be proposed, where the spurious component at $\omega_{o}$ is almost completely cancelled below the noise floor, and the spur rejection level at $3 \omega_{o}$ is about $-29 \mathrm{dBc}$. Comparing the total noise level caused by the spurious tones, we observe that the second case gives close to $3 \mathrm{~dB}$ better inband SNR, making it a preferable option in terms of leakage cancellation. The achieved improvement is desirable, though not necessarily enough. Both spurious tones can further be reduced by filtering, as seen next.

\section{EFFECT OF FILTERING}

Let us assume that we can implement a single resonator filter in the output of each multiply-by-2 stage. Then, the center

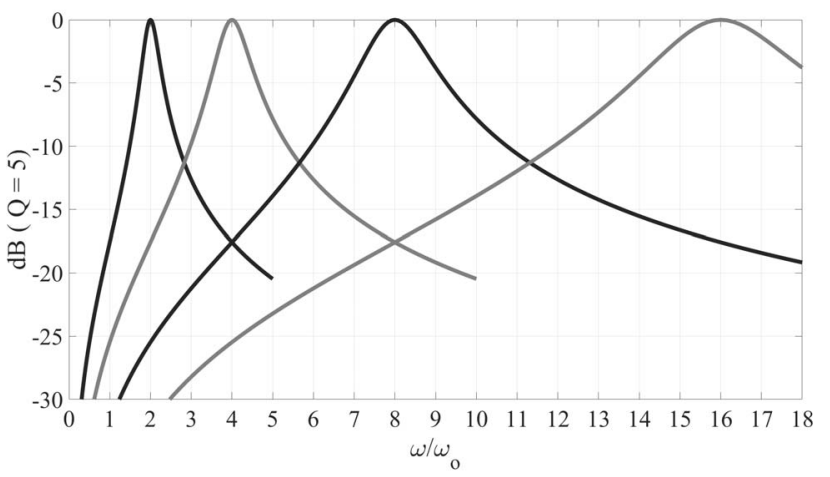

Fig. 8. Frequency response of the filter in different center frequencies $(Q=$ $5)$

frequency of each resonator will be the desired harmonic $n \omega_{o}$ of that particular stage, while the nearest spurious tone $\omega$ to be attenuated will be $(n-1) \omega_{o}$. The achievable cancellation $(A t t)$ can be calculated by substituting $\omega$ into the bandpass response in (8). Alternatively, the required $Q$ for a given attenuation can be calculated using (9)

$$
\begin{gathered}
\frac{1}{A t t}=H_{\text {filter }}(\omega)=\frac{j \omega\left(n \omega_{o} / Q\right)}{n^{2} \omega_{o}^{2}-\omega^{2}+j \omega\left(n \omega_{o} / Q\right)} \\
Q=\frac{1+2 n}{n(n-1)} \sqrt{\left.A t t\right|_{(n-1) \omega_{o}}-1}
\end{gathered}
$$

By substituting a practical $Q$-value of 5 into (8) as shown in Fig. 8, we achieve $-17 \mathrm{~dB}$ of attenuation in the nearest spurious tone at the first stage $(n=2)$, while in the multiplyby-16 output $(n=16)$ the attenuation will be only $-1.5 \mathrm{~dB}$. Hence, the filtering is most effective in the first stage, and quite useless in the last stage.

\section{DISCUSSION}

Based on the above explanations, the generation of close spurious tones, $\left(2^{k}-1\right) \omega_{o}$, in multiply-by- $2^{k}$ signal chains has two main root causes: a) leaking the original LO signal into the first stage in the multiplier chain; b) the presence of all the second-order products in the output whenever there are more than two tones in the input of the doubler.

According to (a), it seems possible to minimize the closest spurious tones by minimizing the LO feedthrough in the first doubler. There are three possible ways of doing that. First, we can cancel the common mode feed-through by adjusting the biasing of the multiplier so that the commonmode fundamental components can be canceled out. Second, the LO feedthrough can also be filtered, which is doable in the first stage where the tones are widely spaced. Third, we can try to minimize the imbalance and common mode generation in the input of the first doubler. This can be done by carefully designing the balun (given the mismatch limit given by (2)). 


\section{REFERENCES}

[1] H. Darabi et al., "A Quad-Band GSM/GPRS/EDGE SoC in $65 \mathrm{~nm}$ CMOS," in IEEE Journal of Solid-State Circuits, vol. 46, no. 4, pp. 870-882, April 2011.

[2] R. B. Staszewski, K. Waheed, F. Dulger and O. E. Eliezer, "Spur-Free Multirate All-Digital PLL for Mobile Phones in $65 \mathrm{~nm}$ CMOS," in IEEE Journal of Solid-State Circuits, vol. 46, no. 12, pp. 2904-2919, Dec. 2011.

[3] Y. Choi, Y. Seong, Y. Yoo, S. Lee, M. Velazquez Lopez and H. Yoo, "Multi-Standard Hybrid PLL With Low Phase-Noise Characteristics for GSM/EDGE and LTE Applications," in IEEE Transactions on Microwave Theory and Techniques, vol. 63, no. 10, pp. 3254-3264, Oct. 2015.

[4] N. Mahalingam, Y. Wang, B. K. Thangarasu, K. Ma and K. S. Yeo, ”A 30-GHz Power-Efficient PLL Frequency Synthesizer for 60-GHz Applications," in IEEE Transactions on Microwave Theory and Techniques, vol. 65, no. 11, pp. 4165-4175, Nov. 2017.

[5] C. Karnfelt, R. Kozhuharov, H. Zirath and I. Angelov, "High-purity 60-GHz-band single-chip $\times 8$ multipliers in pHEMT and mHEMT technology," in IEEE Transactions on Microwave Theory and Techniques, vol. 54, no. 6, pp. 2887-2898, June 2006.

[6] E. Öjefors, B. Heinemann and U. R. Pfeiffer, "A $325 \mathrm{GHz}$ frequency multiplier chain in a SiGe HBT technology," 2010 IEEE Radio Frequency Integrated Circuits Symposium, Anaheim, CA, 2010, pp. 91-94.

[7] E. Ojefors, B. Heinemann and U. R. Pfeiffer, "Active 220- and 325-GHz Frequency Multiplier Chains in an SiGe HBT Technology," in IEEE Transactions on Microwave Theory and Techniques, vol. 59, no. 5, pp. 1311-1318, May 2011.

[8] L. Wang, Y. Xiong, B. Zhang, S. Hu and T. Lim, "Millimeter-Wave Frequency Doubler With Transistor Grounded-Shielding Structure in $0.13-\mu \mathrm{mSiGe}$ BiCMOS Technology," in IEEE Transactions on Microwave Theory and Techniques, vol. 59, no. 5, pp. 1304-1310, May 2011.

[9] N. Sarmah, B. Heinemann and U. R. Pfeiffer, "235-275 GHz (x16) frequency multiplier chains with up to $0 \mathrm{dBm}$ peak output power and low DC power consumption,” 2014 IEEE Radio Frequency Integrated Circuits Symposium, Tampa, FL, 2014, pp. 181-184.

[10] J. Grzyb, K. Statnikov, N. Sarmah, B. Heinemann and U. R. Pfeiffer, "A 210-270-GHz Circularly Polarized FMCW Radar With a Single-LensCoupled SiGe HBT Chip," in IEEE Transactions on Terahertz Science and Technology, vol. 6, no. 6, pp. 771-783, Nov. 2016. 